\title{
On spectral estimates for two-dimensional Schrödinger operators
}

\author{
Ari Laptev and Michael Solomyak
}

\begin{abstract}
For the two-dimensional Schrödinger operator $\mathbf{H}_{\alpha V}=-\Delta-\alpha V, V \geq 0$, we study the behavior of the number $N_{-}\left(\mathbf{H}_{\alpha V}\right)$ of its negative eigenvalues (bound states), as the coupling parameter $\alpha$ tends to infinity. A wide class of potentials is described, for which $N_{-}\left(\mathbf{H}_{\alpha V}\right)$ has the semi-classical behavior, i.e. $N_{-}\left(\mathbf{H}_{\alpha V}\right)=O(\alpha)$. For the potentials from this class, the necessary and sufficient condition is found for the validity of the Weyl asymptotic law.
\end{abstract}

Mathematics Subject Classification (2010). Primary 35J10; Secondary 35P20.

Keywords. Schrödinger operator on $\mathbb{R}^{2}$, bound states, spectral estimates.

\section{Introduction}

1.1. Preliminaries. Let $\mathbf{H}_{\alpha V}$ be a Schrödinger operator

$$
\mathbf{H}_{\alpha V}=-\Delta-\alpha V
$$

on $\mathbb{R}^{2}$. We suppose that $V \geq 0$, and $\alpha>0$ is the coupling constant. We write $N_{-}\left(\mathbf{H}_{\alpha V}\right)$ for the number of negative eigenvalues of $\mathbf{H}_{\alpha V}$, counted with multiplicities:

$$
N_{-}\left(\mathbf{H}_{\alpha V}\right)=\#\left\{j \in \mathbb{N}: \lambda_{j}\left(\mathbf{H}_{\alpha V}\right)<0\right\} \text {. }
$$

As it is well known, the lowest possible (semi-classical) rate of growth of this function is

$$
N_{-}\left(\mathbf{H}_{\alpha V}\right)=O(\alpha), \quad \alpha \rightarrow \infty .
$$

This agrees with the Weyl-type asymptotic formula

$$
\lim _{\alpha \rightarrow \infty} \alpha^{-1} N_{-}\left(\mathbf{H}_{\alpha V}\right)=\frac{1}{4 \pi} \int_{\mathbb{R}^{2}} V d x
$$

that is satisfied if the potential behaves fine enough. 
The exhaustive description of the classes of potentials on $\mathbb{R}^{2}$, such that (1.2) or (1.3) is satisfied, is unknown till now. This is in contrast with the case of dimensions $d>2$, where the celebrated Cwikel-Lieb-Rozenblum estimate describes the class of potentials, for which both the estimate $N_{-}\left(\mathbf{H}_{\alpha V}\right)=O\left(\alpha^{d / 2}\right)$ and the Weyl asymptotic formula hold true.

In the forthcoming discussion, $\mathcal{P}_{\text {semi }}$ stands for the class of all potentials $V \geq 0$ on $\mathbb{R}^{2}$, such that (1.2) is satisfied, and $\mathcal{P}_{\text {Weyl }}$ stands for the class of all such potentials that asymptotics (1.3) holds true. It is clear that

$$
\mathcal{P}_{\text {Weyl }} \subset \mathcal{P}_{\text {semi }} \text {. }
$$

The first results describing wide classes of potentials $V \in \mathcal{P}_{\text {semi }}$ were obtained in [10] and [1]. In the latter paper, this was done also for the class $\mathcal{P}_{\text {Weyl }}$. In particular, it was shown there that the inclusion in (1.4) is proper. What is more, in [1] the general nature of potentials $V \in \mathcal{P}_{\text {semi }} \backslash \mathcal{P}_{\text {Weyl }}$ was explained.

Some further estimates guaranteeing $V \in \mathcal{P}_{\text {semi }}$ were obtained in the recent paper [4]. We would like to mention also the paper [8] whose authors have obtained some new results that give for $N_{-}\left(\mathbf{H}_{\alpha V}\right)$ the order of growth larger than $O(\alpha)$.

In the papers [5] and [3] the important case of radial potentials, $V(x)=F(|x|)$, was analyzed. For such potentials in [3] an integral estimate for $N_{-}\left(\mathbf{H}_{\alpha V}\right)$ was obtained guaranteeing the inclusion $V \in \mathcal{P}_{\text {semi }}$ (actually, it guarantees also that $V \in$ $\left.\mathcal{P}_{\text {Weyl }}\right)$. This result was strengthened in the recent paper [7] where for the radial potentials the necessary and sufficient conditions for $V \in \mathcal{P}_{\text {semi }}$ and for $V \in \mathcal{P}_{\text {Weyl }}$ were established.

In the present paper we return to the study of general (that is, not necessarily radial) potentials. We obtain an estimate that covers the main results of [1] and [4]. It does not cover the estimate obtained in [10], however it has an important advantage compared with the latter: it does not use the intricate Orlicz norms appearing in [10].

1.2. Formulation of the main result. Below $(r, \vartheta)$ stand for the polar coordinates in $\mathbb{R}^{2}$, and $\$$ stands for the unit circle $r=1$. Given a function $V$, such that $V(r, \cdot) \in$ $L_{1}(\$)$ for almost all $r>0$, we introduce its radial and non-radial parts

$$
V_{\mathrm{rad}}(r)=\frac{1}{2 \pi} \int_{\Phi} V(r, \vartheta) d \vartheta ; \quad V_{\mathrm{nrad}}(r, \vartheta)=V(r, \vartheta)-V_{\mathrm{rad}}(r) .
$$

In our result the conditions will be imposed separately on the radial and on the non-radial parts of a given potential $V$. For handling the radial part, we need some auxiliary operator family on the real line, of the form

$$
\left(\mathbf{M}_{\alpha G} \varphi\right)(t)=-\varphi^{\prime \prime}(t)-\alpha G(t) \varphi(t), \quad \varphi(0)=0,
$$

with the "effective potential"

$$
G(t)=G_{V}(t)=e^{2|t|} V_{\mathrm{rad}}\left(e^{t}\right) .
$$


Due to the condition $\varphi(0)=0$ in (1.5), for every $\alpha$ the operator $\mathbf{M}_{\alpha G}$ is the direct sum of two operators, each acting on the half-line. The sharp spectral estimates for $\mathbf{M}_{\alpha G}$ can be given in terms of the number sequence (see eq. (1.13) in [7])

$$
\begin{aligned}
\hat{\mathfrak{z}}(G)=\left\{\widehat{\zeta_{j}}(G)\right\}_{j \geq 0}: \widehat{\zeta_{0}}(G) & =\int_{D_{0}} G(t) d t, \\
\widehat{\zeta_{j}}(G) & =\int_{|t| \in D_{j}}|t| G(t) d t \quad(j \in \mathbb{N})
\end{aligned}
$$

where $D_{0}=(-1,1)$ and $D_{j}=\left(e^{j-1}, e^{j}\right)$ for $j \in \mathbb{N}$. For our purposes, it is convenient to express properties of this sequence in terms of the "weak $\ell_{q}$-spaces" $\ell_{q, \infty}$. Actually, in the main body of this paper we deal only with $q=1$, and below we remind the definition of $\ell_{1, \infty}$. The definition of the weak $\ell_{q}$-spaces with $q \neq 1$ can be found, e.g., in [1], Section 1.4.

Given a sequence of real numbers $\mathbf{x}=\left\{x_{j}\right\}_{j \in \mathbb{N}}$, such that $x_{j} \rightarrow 0$, we denote

$$
n(\varepsilon, \mathbf{x})=\#\left\{j:\left|x_{j}\right|>\varepsilon\right\}, \quad \varepsilon>0 .
$$

The sequence $\mathbf{x}$ belongs to $\ell_{1, \infty}$, if

$$
\|\mathbf{x}\|_{1, \infty} \stackrel{\text { def }}{=} \sup _{\varepsilon>0}(\varepsilon n(\varepsilon, \mathbf{x}))<\infty .
$$

This is a linear space, and the functional $\|\cdot\|_{1, \infty}$ defines a quasinorm in it. The latter means that, instead of the standard triangle inequality, this functional satisfies a weaker property:

$$
\|\mathbf{x}+\mathbf{y}\|_{1, \infty} \leq c\left(\|\mathbf{x}\|_{1, \infty}+\|\mathbf{y}\|_{1, \infty}\right),
$$

with some constant $c>1$ that does not depend on the sequences $\mathbf{x}, \mathbf{y}$. This quasinorm defines a topology in $\ell_{1, \infty}$; there is no norm compatible with this topology.

The space $\ell_{1, \infty}$ is non-separable. Consider its closed subspace $\ell_{1, \infty}^{\circ}$ in which the sequences $\mathbf{x}$ with only a finitely many non-zero terms form a dense subset. This subspace is separable, and its elements are characterized by the property

$$
\mathbf{x} \in \ell_{1, \infty}^{\circ} \Longleftrightarrow \varepsilon n(\varepsilon, \mathbf{x}) \longrightarrow 0, \quad \varepsilon \rightarrow 0 .
$$

The (non-linear) functionals

$$
\Delta_{1}(\mathbf{x})=\limsup _{\varepsilon \rightarrow 0}(\varepsilon n(\varepsilon, \mathbf{x})), \quad \delta_{1}(\mathbf{x})=\liminf _{\varepsilon \rightarrow 0}(\varepsilon n(\varepsilon, \mathbf{x}))
$$

are well-defined on the space $\ell_{1, \infty}$, and

$$
\delta_{1}(\mathbf{x}) \leq \Delta_{1}(\mathbf{x}) \leq\|\mathbf{x}\|_{1, \infty} .
$$

It is clear that $\ell_{1, \infty}^{\circ}=\left\{\mathbf{x} \in \ell_{1, \infty}: \Delta_{1}(\mathbf{x})=0\right\}$. 
The conditions on $V_{\text {nrad }}$ will be given in terms of the space $L_{1}\left(\mathbb{R}_{+}, L_{p}(\$)\right)$, with an arbitrarily chosen $p>1$. This is the function space on $\mathbb{R}^{2}$, with the following norm:

$$
\|f\|_{L_{1}\left(\mathbb{R}_{+}, L_{p}(\Phi)\right)}=\int_{\mathbb{R}_{+}}\left(\int_{\mathbb{\Phi}}|f(r, \vartheta)|^{p} d \vartheta\right)^{1 / p} r d r .
$$

This is a separable Banach space, and the bounded functions whose support is a compact subset in $\mathbb{R}^{2} \backslash\{0\}$ are dense in it. The space $L_{1}\left(\mathbb{R}_{+}, L_{p}(\mathbb{\$})\right)$ was used in the paper [6], and its results are one of the basic tools in our proof below.

Here is the main result of the paper.

Theorem 1.1. Let a potential $V \geq 0$ be such that $\hat{\mathfrak{z}}\left(G_{V}\right) \in \ell_{1, \infty}$, and

$$
V_{\text {nrad }} \in L_{1}\left(\mathbb{R}_{+}, L_{p}(\$)\right) \text { with some } p>1 \text {. }
$$

Then $V \in \mathcal{P}_{\text {semi }}$, and the estimate is satisfied

$$
N_{-}\left(\mathbf{H}_{\alpha V}\right) \leq 1+C(p)\left(\left\|V_{\text {nrad }}\right\|_{L_{1}\left(\mathbb{R}_{+}, L_{p}(\$)\right)}+\left\|\hat{\mathfrak{z}}\left(G_{V}\right)\right\|_{\ell_{1, \infty}}\right) .
$$

Moreover, the following equalities hold true:

$$
\left\{\begin{array}{l}
\limsup _{\alpha \rightarrow \infty} \alpha^{-1} N_{-}\left(\mathbf{H}_{\alpha V}\right)=\frac{1}{4 \pi} \int V d x+\limsup _{\alpha \rightarrow \infty} \alpha^{-1} N_{-}\left(\mathbf{M}_{\alpha} G_{V}\right), \\
\liminf _{\alpha \rightarrow \infty} \alpha^{-1} N_{-}\left(\mathbf{H}_{\alpha V}\right)=\frac{1}{4 \pi} \int V d x+\liminf _{\alpha \rightarrow \infty} \alpha^{-1} N_{-}\left(\mathbf{M}_{\alpha G_{V}}\right) .
\end{array}\right.
$$

In particular, under assumption (1.10) the condition $\hat{\mathfrak{z}}\left(G_{V}\right) \in \ell_{1, \infty}^{\circ}$ is necessary and sufficient for $V \in \mathcal{P}_{\text {Weyl }}$.

In (1.12), and later on, the integral with no domain specified always means $\int_{\mathbb{R}^{2}}$.

Formula (1.12), and especially, its proof in Subsection 3.3, show that, in a certain sense, the parts $V_{\text {rad }}$ and $V_{\text {nrad }}$ contribute to the asymptotic behavior of $N_{-}\left(\mathbf{H}_{\alpha V}\right)$ independently. It may also happen that the contribution of $V_{\text {rad }}$ is stronger than that of $V_{\text {nrad }}$, and "screens" the latter. This situation is described by the following statement, that complements our main theorem.

Proposition 1.2. Let a potential $V \geq 0$ be such that $\hat{\mathfrak{z}}\left(G_{V}\right) \in \ell_{q, \infty}$ with some $q>1$, and (1.10) is satisfied. Then

$$
\left\{\begin{array}{l}
\limsup _{\alpha \rightarrow \infty} \alpha^{-q} N_{-}\left(\mathbf{H}_{\alpha V}\right)=\limsup _{\alpha \rightarrow \infty} \alpha^{-q} N_{-}\left(\mathbf{M}_{\alpha G_{V}}\right), \\
\liminf _{\alpha \rightarrow \infty} \alpha^{-q} N_{-}\left(\mathbf{H}_{\alpha V}\right)=\liminf _{\alpha \rightarrow \infty} \alpha^{-q} N_{-}\left(\mathbf{M}_{\alpha G_{V}}\right) .
\end{array}\right.
$$

This is an analog of statement (b) in Theorem 5.1 of the paper [1]. Its proof is basically the same, and we do not reproduce it here. In the same paper one finds also examples that illustrate the situation described by Proposition 1.2. 


\section{Auxiliary material}

The proof of Theorem 1.1 mainly follows the line worked out in [1] and [10]. The same approach was used in [7], and the material below, in part, duplicates the contents of its Section 2. We systematically use the variational description of the spectrum. In particular, we often define a self-adjoint operator via its corresponding Rayleigh quotient.

2.1. Classes $\Sigma_{\mathbf{1}}, \boldsymbol{\Sigma}_{\mathbf{1}}^{\circ}$ of compact operators. If $\mathbf{T}$ is a linear compact operator in a Hilbert space, then, as usual, $\left\{s_{j}(\mathbf{T})\right\}$ stands for the sequence of its singular numbers, i.e. for the eigenvalues of the non-negative, self-adjoint operator $\left(\mathbf{T}^{*} \mathbf{T}\right)^{1 / 2}$. By $n(\varepsilon, \mathbf{T})$ we denote the distribution function of the singular numbers,

$$
n(\varepsilon, \mathbf{T})=\#\left\{j: s_{j}>\varepsilon\right\}, \quad \varepsilon>0 .
$$

We say that $\mathbf{T}$ belongs to the class $\Sigma_{1}$ if and only if $\left\{s_{j}(\mathbf{T})\right\} \in \ell_{1, \infty}$, and to the class $\Sigma_{1}^{\circ}$ if and only if $\left\{s_{j}(\mathbf{T})\right\} \in \ell_{1, \infty}^{\circ}$. These are linear, quasinormed spaces with respect to the quasinorm $\|\mathbf{T}\|_{1, \infty}$ induced by this definition. The space $\Sigma_{1}$ is nonseparable, and $\Sigma_{1}^{\circ}$ is its separable subspace in which the finite rank operators form a dense subset. Similarly to (1.8), we define the functionals

$$
\Delta_{1}(\mathbf{T})=\Delta_{1}\left(\left\{s_{j}(\mathbf{T})\right\}\right), \quad \delta_{1}(\mathbf{T})=\delta_{1}\left(\left\{s_{j}(\mathbf{T})\right\}\right) .
$$

Note that

$$
\delta_{1}(\mathbf{T}) \leq \Delta_{1}(\mathbf{T}) \leq\|\mathbf{T}\|_{1, \infty} .
$$

See [2], Section 11.6, for more detail about these spaces, and about similar spaces $\Sigma_{q}, \Sigma_{q}^{\circ}$ for any $q>0$.

2.2. Reduction of the main problem to compact operators. Let us introduce two subspaces in $C_{0}^{\infty}\left(\mathbb{R}^{2}\right)$ :

$$
\begin{aligned}
& \mathcal{F}_{0}=\left\{f \in C_{0}^{\infty}: f(x)=\varphi(r), \varphi(1)=0\right\}, \\
& \mathcal{F}_{1}=\left\{f \in C_{0}^{\infty}: \int_{0}^{2 \pi} f(r, \vartheta) d \vartheta=0, \quad r>0\right\} .
\end{aligned}
$$

They are orthogonal to each other both in the $L_{2}$-metric and in the metric of the Dirichlet integral. The Hardy inequalities have a different form on $\mathscr{F}_{0}$ and on $\mathscr{F}_{1}$ :

$$
\begin{gathered}
\int \frac{|f(x)|^{2}}{|x|^{2} \ln ^{2}|x|} d x \leq \frac{1}{4} \int|\nabla f(x)|^{2} d x, \quad f \in \mathscr{F}_{0} ; \\
\int \frac{|f(x)|^{2}}{|x|^{2}} d x \leq \int|\nabla f(x)|^{2} d x, \quad f \in \mathscr{F}_{1} .
\end{gathered}
$$


For proving (2.1), one substitutes $r=|x|=e^{t}$, and then applies the standard Hardy inequality in dimension 1. The proof of (2.2) is quite elementary, it can be found, e.g., in [10], or in [1].

Let us consider the completions $\mathscr{H}_{0}^{1}, \mathscr{H}_{1}^{1}$ of the spaces $\mathcal{F}_{0}, \mathcal{F}_{1}$ in the metric of the Dirichlet integral. It follows from Hardy inequalities (2.1) and (2.2) that these are Hilbert function spaces, embedded into the weighted $L_{2}$, with the weights defined by these inequalities. Consider also their orthogonal sum

$$
\mathscr{H}^{1}=\mathscr{H}_{0}^{1} \oplus \mathscr{H}_{1}^{1} \text {. }
$$

An independent definition of this Hilbert space is

$$
\mathscr{H}^{1}=\left\{f \in H_{\mathrm{loc}}^{1}\left(\mathbb{R}^{2}\right): \int_{0}^{2 \pi} f(1, \vartheta) d \vartheta=0,|\nabla f| \in L_{2}\left(\mathbb{R}^{2}\right)\right\},
$$

with the metric of the Dirichlet integral.

We also define the spaces $H_{0}^{1}, H_{1}^{1}$ which are the completions of $\mathcal{F}_{0}, \mathcal{F}_{1}$ in $H^{1}\left(\mathbb{R}^{2}\right)$, and

$$
\tilde{H}^{1}=H_{0}^{1} \oplus H_{1}^{1}=\left\{f \in H^{1}\left(\mathbb{R}^{2}\right): \int_{0}^{2 \pi} f(1, \vartheta) d \vartheta=0\right\} .
$$

This is a subspace in $H^{1}\left(\mathbb{R}^{2}\right)$ of codimension 1 .

Finally, we need the spaces $\mathscr{E}_{0}, \mathscr{E}_{1}$ which are the completions of $\mathcal{F}_{0}, \mathcal{F}_{1}$ in the $L_{2}$-metric. Note that the condition $\varphi(1)=0$, occurring in the description of $\mathcal{F}_{0}$, disappears for general $f \in \mathscr{E}_{0}$.

Suppose that $V \geq 0$ is a measurable function, such that

$$
\mathbf{b}_{V}[u] \stackrel{\text { def }}{=} \int V|u|^{2} d x \leq C \int|\nabla u|^{2} d x, \quad u \in \mathscr{H}^{1} .
$$

Under assumption (2.4) the quadratic form $\mathbf{b}_{V}$ defines a bounded self-adjoint operator $\mathbf{B}_{V} \geq 0$ in $\mathscr{H}^{1}$. If (and only if) this operator is compact, then, by the BirmanSchwinger principle, the quadratic form

$$
\int\left(|\nabla u|^{2}-\alpha V|u|^{2}\right) d x
$$

with the form-domain $\tilde{H}^{1}$ is closed and bounded from below for each $\alpha>0$, the negative spectrum of the associated self-adjoint operator $\widetilde{\mathbf{H}}_{\alpha V}$ on $L_{2}\left(\mathbb{R}^{2}\right)$ is finite, and the following equality for the number of its negative eigenvalues holds true:

$$
N_{-}\left(\tilde{\mathbf{H}}_{\alpha V}\right)=n\left(\alpha^{-1}, \mathbf{B}_{V}\right), \quad \alpha>0 .
$$

Now, let us withdraw the rank one condition $\int_{0}^{2 \pi} u(1, \vartheta) d \vartheta=0$ from the description of the form-domain. Then the resulting quadratic form corresponds to the Schrödinger operator $\mathbf{H}_{\alpha V}$ as in (1.1). Hence,

$$
N_{-}\left(\tilde{\mathbf{H}}_{\alpha V}\right) \leq N_{-}\left(\mathbf{H}_{\alpha V}\right) \leq N_{-}\left(\tilde{\mathbf{H}}_{\alpha V}\right)+1,
$$


and, by (2.6),

$$
n\left(\alpha^{-1}, \mathbf{B}_{V}\right) \leq N_{-}\left(\mathbf{H}_{\alpha V}\right) \leq n\left(\alpha^{-1}, \mathbf{B}_{V}\right)+1 .
$$

Thus, the study of the quantity $N_{-}\left(\mathbf{H}_{\alpha V}\right)$ for all $\alpha>0$ is reduced to the investigation of the "individual" operator $\mathbf{B}_{V}$, which is nothing but the Birman-Schwinger operator for the family of operators in $L_{2}\left(\mathbb{R}^{2}\right)$ associated with the family of quadratic forms in (2.5). Note that the Birman-Schwinger operator for the original family in (1.1) is ill-defined, since the completion of the space $H^{1}\left(\mathbb{R}^{2}\right)$ in the metric of the Dirichlet integral is not a space of functions on $\mathbb{R}^{2}$.

\section{Proof of Theorem 1.1}

3.1. Decomposition of the quadratic form $\mathbf{b}_{\boldsymbol{V}}$.. Given a function $u \in \mathscr{H}^{1}$, we agree to standardly denote its components in decomposition $(2.3)$ by $\varphi(r), v(r, \vartheta)$. Along with the quadratic form $\mathbf{b}_{V}$, we consider its "parts" in the subspaces $\mathscr{H}_{0}^{1}, \mathscr{H}_{1}^{1}$ :

$$
\mathbf{b}_{V, 0}[u]=\mathbf{b}_{V}[\varphi], \quad \mathbf{b}_{V, 1}[u]=\mathbf{b}_{V}[v] .
$$

Let $\mathbf{B}_{V, j}, j=0,1$, stand for the corresponding self-adjoint operators in $\mathscr{H}_{j}^{1}$. Using orthogonal decomposition (2.3), we see that

$$
\mathbf{b}_{V}[u]=\mathbf{b}_{V, 0}[\varphi]+\mathbf{b}_{V, 1}[v]+2 \int V(x) \operatorname{Re}(\varphi(|x|) \overline{v(x)}) d x .
$$

For the radial potentials the last term vanishes, and this considerably simplifies the reasoning, see [7]. For the general potentials this is no more true. Still, the following inequality is always valid:

$$
\mathbf{b}_{V}[u] \leq 2\left(\mathbf{b}_{V}[\varphi]+\mathbf{b}_{V}[v]\right)
$$

and it shows that for estimation of $\left\|\mathbf{B}_{V}\right\|_{1, \infty}$ it suffices to evaluate the quasinorms in $\Sigma_{1}$ of the operators $\mathbf{B}_{V, 0}, \mathbf{B}_{V, 1}$ separately.

The estimation of $\left\|\mathbf{B}_{V, 0}\right\|_{1, \infty}$ will be based upon the following result on the operators $\mathbf{F}_{G}$ on real line, whose Rayleigh quotient is

$$
\frac{\int_{\mathbb{R}} G(t)|\omega(t)|^{2} d t}{\int_{\mathbb{R}}\left|\omega^{\prime}(t)\right|^{2} d t}, \quad \omega(0)=0 .
$$

Clearly, this is the Birman-Schwinger operator for the family $\mathbf{M}_{\alpha G}$ given by (1.5).

Proposition 3.1. Let a function $G \in L_{1, l o c}(\mathbb{R}), G \geq 0$, be given. Define the corresponding number sequence $\hat{\mathfrak{z}}(G)$ as in $(1.7)$, and suppose that $\hat{\mathfrak{z}}(G) \in \ell_{1, \infty}$. 
Then the operator $\mathbf{F}_{G}$ is well-defined, belongs to the class $\Sigma_{1}$, and the estimate is satisfied,

$$
\left\|\mathbf{F}_{G}\right\|_{1, \infty} \leq C\|\hat{\mathfrak{z}}(G)\|_{1, \infty} .
$$

If $\hat{\mathfrak{z}}(G) \in \ell_{1, \infty}^{\circ}$, then $\hat{\mathfrak{z}}(G) \in \Sigma_{1}^{\circ}$.

For the proof, see Section 4 in the paper [1]. There the operators on the half-line were considered, however the passage to the case of the whole line is straightforward, due to the condition $\omega(0)=0$ in (3.3). In this respect, see also a discussion in [7], Section 3.

Now we turn to the operator $\mathbf{B}_{V, 1}$. The estimation of its quasinorm in $\Sigma_{1}$ uses a result that is a particular case (for $l=1$ ) of Theorem 1.2 in the paper [6]. We present its equivalent formulation, more convenient for our purposes. Namely, we formulate it for the Birman-Schwinger operator, rather than for the original Schrödinger operator, as it was done in [6].

Proposition 3.2. Let $V \geq 0, V \in L_{1}\left(\mathbb{R}_{+}, L_{p}(\$)\right)$, with some $p>1$. Then the operator $\widehat{\mathbf{B}}_{V}$, whose Rayleigh quotient is

$$
\frac{\int V(x)|u|^{2} d x}{\int\left(|\nabla u|^{2}+|x|^{-2}|u|^{2}\right) d x}, \quad u \in \mathscr{H}_{1}^{1},
$$

belongs to the class $\Sigma_{1}$, and

$$
\left\|\widehat{\mathbf{B}}_{V}\right\|_{1, \infty} \leq C(p)\|V\|_{L_{1}\left(\mathbb{R}_{+}, L_{p}(\$)\right)} .
$$

We recall that the norm appearing in (3.6) was defined in (1.9).

3.2. Proof of (1.11). As it was explained in the previous subsection, we have to estimate the quasinorms of the operators $\mathbf{B}_{V, 0}, \mathbf{B}_{V, 1}$ in the space $\Sigma_{1}$.

Consider first the operator $\mathbf{B}_{V, 0}$. The corresponding Rayleigh quotient is

$$
\frac{\int_{\mathbb{R}^{2}} V(r, \vartheta)|\varphi(r)|^{2} r d r d \vartheta}{\int_{\mathbb{R}^{2}}\left|\varphi^{\prime}(r)\right|^{2} r d r d \vartheta}=\frac{\int_{0}^{\infty} V_{\mathrm{rad}}(r)|\varphi(r)|^{2} r d r}{\int_{0}^{\infty}\left|\varphi^{\prime}(r)\right|^{2} r d r} .
$$

The standard substitution $r=e^{t}, \varphi(r)=\omega(t) ; t \in \mathbb{R}$, reduces it to the form

$$
\frac{\int_{\mathbb{R}} G_{V}(t)|\omega(t)|^{2} d t}{\int_{\mathbb{R}}\left|\omega^{\prime}(t)\right|^{2} d t}, \quad \omega(0)=0 .
$$


where the potential $G_{V}$ is given by (1.6). Now, Proposition 3.1 applies, and we arrive at the estimate

$$
\left\|\mathbf{B}_{V, 0}\right\|_{1, \infty} \leq C\|\hat{\mathfrak{z}}(G)\|_{1, \infty} .
$$

The Rayleigh quotient for the operator $\mathbf{B}_{V, 1}$ is given by

$$
\frac{\int V(x)|u|^{2} d x}{\int|\nabla u|^{2} d x}, \quad u \in \mathscr{H}_{1}^{1} .
$$

Due to Hardy inequality (2.2), on the subspace $\mathscr{H}_{1}^{1}$ the norm of the Dirichlet integral is equivalent to the norm generated by the quadratic form in the denominator of (3.5). Hence, estimate (3.6) applies to this operator, with some other constant factor $C^{\prime}(p)$. So, we have

$$
\left\|\mathbf{B}_{V, 1}\right\|_{1, \infty} \leq C^{\prime}(p)\|V\|_{L_{1}\left(\mathbb{R}_{+}, L_{p}(\Phi)\right)} .
$$

Estimates (3.4) and (3.8), together with inequality (3.2), imply the desired (1.11).

3.3. Proof of (1.12). First of all, we are going to show that

$$
\lim _{\varepsilon \rightarrow 0}\left(\varepsilon n\left(\varepsilon, \mathbf{B}_{V, 1}\right)\right)=\frac{1}{4 \pi} \int_{\mathbb{R}^{2}} V d x .
$$

For $V \in C_{0}^{\infty}\left(\mathbb{R}^{2} \backslash\{0\}\right)$, Theorem 5.1 in [1] yields that

$$
\mathbb{N}_{-}\left(\mathbf{H}_{\alpha V}\right) \sim(4 \pi)^{-1} \alpha \int V d x, \quad \alpha \rightarrow \infty .
$$

By the Birman-Schwinger principle, this is equivalent to

$$
n\left(\varepsilon, \mathbf{B}_{V}\right) \sim(4 \pi \varepsilon)^{-1} \int V d x, \quad \varepsilon \rightarrow 0 .
$$

The spectrum of $\mathbf{B}_{V, 1}$ has the same asymptotic behavior, since for such potentials the subspace $\mathscr{H}_{0}^{1}$ does not contribute to the asymptotic coefficient.

Now, let $V \geq 0$ be an arbitrary potential from $L_{1}\left(\mathbb{R}_{+}, L_{p}(\$)\right)$. Then, approximating it by the functions from $C_{0}^{\infty}$ and taking into account the continuity of the asymptotic coefficients in the metric of $\Sigma_{1}$ (see [2], Theorem 11.6.6), we extend the formula to all such $V$. So, (3.9) is established.

Return to the study of the operator $\mathbf{B}_{V}$. Along with it, let us consider the direct orthogonal sum $\mathscr{B}_{V}=\mathbf{B}_{V, 0} \oplus \mathbf{B}_{V, 1}$. Evidently,

$$
n\left(\varepsilon, \mathscr{B}_{V}\right)=n\left(\varepsilon, \mathbf{B}_{V, 0}\right)+n\left(\varepsilon, \mathbf{B}_{V, 1}\right) .
$$


Hence, for justifying asymptotic formulae (1.12) it suffices to show that the offdiagonal term in (3.1) generates an operator of the class $\Sigma_{1}^{\circ}$. To this end, we first of all note that

$$
\int V \operatorname{Re}(\varphi \bar{v}) d x=\int V_{\text {nrad }} \operatorname{Re}(\varphi \bar{v}) d x,
$$

since $v$ is orthogonal (in $L_{2}$ ) to all functions depending only on $|x|$.

Suppose now that the function $V_{\text {nrad }}$ has a compact support in $\mathbb{R}^{2} \backslash\{0\}$. Then the integral in the right-hand side of (3.10) is actually taken over some annulus $a \leq r \leq a^{-1}, a<1$. Hence,

$$
\begin{aligned}
2\left|\int V_{\text {nrad }} \operatorname{Re}(\varphi \bar{v}) d x\right| \leq & \delta \int_{a}^{a^{-1}} r d r \int_{\Phi}\left|V_{\text {nrad }}(r, \vartheta)\right||v(r, \vartheta)|^{2} d \vartheta \\
& +\delta^{-1} \int_{a}^{a^{-1}} r d r \int_{\Phi}\left|V_{\text {nrad }}(r, \vartheta)\right||\varphi(r)|^{2} d \vartheta .
\end{aligned}
$$

The first term on the right generates an operator on $\mathscr{H}_{1}^{1}$, say, $\mathbf{T}_{1}$, to which estimate (3.8) applies, and it gives

$$
\left\|\mathbf{T}_{1}\right\|_{1, \infty} \leq C^{\prime}(p) \delta .
$$

The second term generates an operator on $\mathscr{H}_{0}^{1}$, say, $\mathbf{T}_{0}$. Its Rayleigh quotient is of the (3.7) but with the integration over a compact subset in $(0, \infty)$. It follows that the spectrum of $\mathbf{T}_{0}$ obeys Weyl's asymptotic law, $\lambda_{j}\left(\mathbf{T}_{0}\right) \asymp c^{-2}$, and hence, $\mathbf{T}_{0} \in \Sigma_{1}^{\circ}$. Taking $\delta$ arbitrarily small, we conclude that asymptotics (1.12) is satisfied in the case where $V_{\text {nrad }}$ is compactly supported.

Finally, we approximate the function $V_{\text {nrad }}$ by compactly supported functions in metric (1.9), and again apply Theorem 11.6.5 from the book [2]. This extends asymptotic formula (1.12) to all potentials, that meet the conditions of Theorem 1.1, and thus, concludes the proof.

Added in Proof. Estimate (1.11) can be replaced by a stronger estimate

$$
N_{-}\left(\mathbf{H}_{\alpha V}\right) \leq 1+C(p) \alpha\left(\left\|V_{\text {nrad }}\right\|_{L_{1}\left(\mathbb{R}_{+}, \mathcal{B}(\Phi)\right)}+\left\|\hat{\mathfrak{z}}\left(G_{V}\right)\right\|_{\ell_{1, \infty}}\right),
$$

where $\mathcal{B}\left(S_{q}\right)$ is the Orlicz space $L \log L$ on the unit circle. This improvement became possible due to the recent result of Shargorodsky [9] (see Section 6 there). 


\section{References}

[1] M. Sh. Birman and A. Laptev, The negative discrete spectrum of a two-dimensional Schrödinger operator. Comm. Pure Appl. Math. 49 (1996), 967-997. MR 1399202 Zbl 0864.35080

[2] M. Sh. Birman and M. Solomyak, Spectral theory of self-adjoint operators in Hilbert space. Translation from the 1980 Russian original by S. Khrushchev and V. Peller. Mathematics and Its Applications. Soviet Series 5. D. Reidel Publishing Co. (Kluwer Academic Publishers), Dordrecht etc., 1987. MR 1192782 Zbl 0744.47017

[3] K. Chadan, N. N. Khuri, A. Martin, and T. T. Wu, Bound states in one and two spatial dimensions. J. Math. Phys. 44 (2003), 406-422. MR 1952194 Zbl 1061.81072

[4] A. Grigor'yan and N. Nadirashvili, Negative eigenvalues of two-dimensional Schrödinger operators. Preprint 2011. arXiv:1112.4986

[5] A. Laptev, The negative spectrum of a class of two-dimensional Schrdinger operators with potentials depending only on radius. Funktsional. Anal. i Prilozhen. 34 (2000), 85-87. English transl.: Funct. Anal. Appl. 34 (2000), 305-307. MR 1819649 Zbl 0972.35079

[6] A. Laptev and Yu. Netrusov, On the negative eigenvalues of a class of Schrödinger operators. In V. Buslaev, M. Solomyak, and D. Yafaev (eds), Differential operators and spectral theory. M. Sh. Birman's $70^{\text {th }}$-anniversary collection. American Mathematical Society Translations, Series 2, 189. Advances in the Mathematical Sciences 41. Amer. Math. Soc., Providence, R.I., 1999, 173-186. MR 1730512 Zbl 0941.35055

[7] A. Laptev and M. Solomyak, On the negative spectrum of the two-dimensional Schrödinger operator with radial potential. Comm. Math. Phys. 314 (2012), 229-241. MR 2954515 Zbl 1253.35037

[8] S. Molchanov and B. Vainberg, On negative eigenvalues of low-dimensional Schrödinger operators. Preprint 2011. arXiv:1105.0937

[9] E. Shargorodsky, On negative eigenvalues of two-dimensional Schrödinger operators. Preprint 2012. arXiv:1203.4833

[10] M. Solomyak, Piecewise-polynomial approximation of functions from $H^{\ell}\left((0,1)^{d}\right)$, $2 \ell=d$, and applications to the spectral theory of the Schrödinger operator. Israel J. Math. 86 (1994), 253-275. MR 1276138 Zbl 0803.47045

Received February 5, 2012; revised October 31, 2012

Ari Laptev, Department of Mathematics, Imperial College London, Huxley Building, 180 Queen's Gate, London SW7 2AZ, U.K.

E-mail: a.laptev@imperial.ac.uk

Michael Solomyak, Department of Mathematics, Weizmann Institute, Rehovot, Israel

E-mail: michail.solomyak@weizmann.ac.il 